\title{
reviscafuences
}

ISSN: 1575-7072 | e-ISSN: 2172-7775

Páginas: 105-126

Recibido: 2019-06-04

Revisado: 2020-02-17

Aceptado: 2020-04-01

Preprint: 2020-04-20

Publicación Final: 2020-06-15 www.revistascientificas.us.es/index.php/fuentes/index

DOI: https://doi.org/10.12795/revistafuentes.2020.v22.i1.09

\section{Factores que inciden en la deserción estudiantil en carreras de perfil Ingeniería Informática}

\section{Factors that affect student desertion in careers in Computer Engineering profile}

\author{
(iD) Niurys Lázaro Alvarez \\ Universidad de las Ciencias Informáticas (Cuba) \\ Zoraida Callejas \\ Universidad de Granada (España) \\ David Griol \\ Universidad Carlos III de Madrid (España)
}

\section{Resumen}

El propósito de esta investigación es identificar los factores relevantes que inciden en la deserción de los estudiantes universitarios, en particular en el contexto de carreras del perfil Ingeniería Informática en la Educación Superior cubana. Se analizan investigaciones previas en el área y estudios específicos de deserción en las enseñanzas técnicas. Posteriormente se realiza un estudio cuantitativo para detectar los factores predictivos de deserción en una muestra de 485 estudiantes de una cohorte de nuevo ingreso de todas las provincias del país, utilizando análisis de correlación, regresión logística y análisis discriminante. Los resultados indican que las variables provincia de procedencia, la opción en que solicitó la carrera, la nota del examen de ingreso en Matemática y el rendimiento académico en Matemática y Programación, son factores incidentes en la deserción estudiantil en el primer año de carreras de perfil Ingeniería Informática. Se obtiene una función discriminante capaz de clasificar correctamente el $91.5 \%$ de los casos estudiados. Estos resultados son importantes para la identificación de estudiantes en riesgo de deserción y la realización de propuestas pedagógicas que los atiendan.

\section{Abstract}

The purpose of this piece of research is to identify the relevant factors that affect the dropout of university students, particularly in the context of careers in the Computer Engineering profile in Cuban Higher Education. Previous research in the area and specific dropout studies in the technical studies are analyzed. Subsequently, a quantitative study is carried out to detect the predictive factors of dropout in a sample of 485 students from a cohort of all the provinces of the country, using correlation analysis, logistic regression and discriminant analysis. The results indicate that the variables province of origin, the option in which the degree was requested, the grades obtained for the entrance examination in Mathematics and the academic performance in Mathematics and Programming, are influential factors in student dropout in the first year of Computer Engineering. A discriminant function is obtained that accurately classifies $91.5 \%$ of cases. These results are important to identify students at risk of dropout and proposing pedagogical strategies to help them.
\end{abstract}

\section{Palabras clave / Keywords}

abandono; retención estudiantil; educación superior; ingeniería informática.

drop-out; student retention; higher education; computer engineering. 


\section{Introducción}

La deserción de los estudiantes en la Educación Superior (ES) es una problemática multifactorial, que afecta, en mayor o menor medida, a todas las instituciones de ES. Ésta reviste gran importancia no sólo para los propios estudiantes e instituciones educativas, sino también para el ámbito empresarial y las oportunidades laborales en cada región, en particular para profesiones que demandan un gran número de trabajadores con una alta preparación.

La demanda de graduados en las ramas de Ciencia, Tecnología, Ingeniería y Matemáticas (STEM, por sus siglas en inglés) ha experimentado un incremento debido al creciente desarrollo científico-tecnológico y sus aplicaciones. Sin embargo, las estadísticas recientes muestran que la deserción en estas carreras es alta (Peña-Calvo, Inda-Caro, Rodríguez-Menéndez, y Fernández-García, 2016; Soistak, Martins, y Galan, 2018). En 2015 se produjo en Europa un crecimiento de un 13\% en la demanda de ingenieros y científicos y se prevé que esta demanda se incremente en un $14 \%$ para 2020 , esto contrasta con el hecho de que la matrícula de estudiantes de los grados de Ingeniería y Arquitectura disminuyó en un $24.6 \%$ del 2004 al 2014 en este mismo contexto, lo que implica que uno de cada cuatro estudiantes ha dejado de estudiar estos grados (López, Carpeño, Arriaga, y Ruiz, 2016).

En el contexto latinoamericano, la Oficina Regional de Educación para América Latina y el Caribe (OREALC) declara que, en la región, sólo 1 de cada 10 jóvenes de 25 a 29 años había completado cinco años de ES en 2010 (Bellei, 2013: 136). Se evidencia por tanto la necesidad de identificar factores predictivos de deserción que puedan atenderse para garantizar su permanencia.

El conocimiento de las causas de deserción debe partir de las propias instituciones y contextos específicos para poder actuar en consecuencia y dirigir acciones institucionales para paliarlas (Tinto, 2010). Asimismo, desde el proyecto GUIA se recomienda continuar identificando las formas en que se presenta la deserción no sólo de manera general, sino también en los distintos contextos particulares donde ocurre, pues es susceptible o permeable a los cambios culturales y personales (Arriaga, Velásquez, \& Coord., 2013). Incluso en el marco del III Congreso Educativo INACAP 2015, Tinto destacó la importancia de desarrollar políticas de retención desde las instituciones educativas específicas atendiendo a sus particularidades (INACAP, 2015).

En tal sentido, el propósito de esta investigación es identificar los factores predictivos que llevan a la deserción de los estudiantes en grados del perfil Ingeniería Informática en Cuba. Existen dos carreras con este perfil en la ES cubana: Ingeniería Informática e Ingeniería en Ciencias Informáticas; el estudio se ha realizado mediante una metodología cuantitativa.

Para cumplir con el propósito de la investigación se plantearon los siguientes objetivos:

- Realizar un análisis detallado de los modelos iniciales de deserción/retención estudiantil en ES y en particular en las carreras de ingeniería informática a través de la revisión de la bibliografía que aborda la temática.

- Plantear y validar un conjunto de hipótesis de investigación que relacionen factores de deserción previos y posteriores al ingreso de los estudiantes con la deserción en las carreras de perfil de Ingeniería Informática en Cuba.

El artículo se continúa desarrollando en seis apartados, en el apartado 2 se analizan los enfoques y variables de los modelos iniciales de deserción/retención estudiantil en la ES estudiados; en el apartado 3 se particulariza los estudios de deserción en carreras de Ingeniería Informática. En el apartado 4 se plantean las hipótesis de investigación y se describen la muestra, variables y el estudio cuantitativo realizado mediante la correlación, regresión logística y análisis discriminante. El apartado 5 presenta y analiza los resultados obtenidos en el análisis descriptivo, correlacional y predictivo. Por cuestiones de espacio, solo se describe un resumen de los resultados de la regresión y análisis discriminante. Por último se plantean las conclusiones más importantes a las que llegamos y las proyecciones futuras.

\section{Modelos de deserción/retención estudiantil universitaria}

Los primeros modelos de deserción-retención estudiantil en instituciones de ES datan de 1970 (Spady, 1970). Existen diferentes enfoques para su introducción, tales como: psicológico, sociológico, interaccionista, organizacional y economicista. En los estudios para explicar la problemática, se consideran variables personales, familiares, institucionales y académicas (Donoso y Schiefelbein, 2007). 
El modelo de Spady (1970) tiene un enfoque sociológico y considera que la integración social en la Universidad determina el compromiso y la decisión de abandonar del estudiante y que los antecedentes familiares son los que determinan el potencial y desempeño académico, junto a las normativas y apoyo de pares.

En los enfoques interaccionista y organizacional se destaca el modelo de Tinto (1975) que tiene en cuenta la integración del estudiante a la Universidad a partir de su interacción académica y social. Concibe que la decisión de abandonar se produce a partir de la relación que se establece entre el resultado de la interacción social y académica que tenga el estudiante durante sus estudios superiores y los propósitos, metas y compromiso con la institución. Con este mismo enfoque también se introducen los modelos de (Bean, 1980; Pascarella \& Terenzini, 1980). Bean considera factores académicos, psicosociales y ambientales para la integración y adaptación a la Universidad, se centra más en la satisfacción del estudiante con la institución; mientras Pascarela y Terenzini utilizan elementos de los dos anteriores y le adicionan la calidad del esfuerzo percibido, dando una gran relevancia a las características organizativas y al ambiente de la Institución.

El modelo de (Ethington, 1990) tiene un enfoque psicológico donde se incorporan variables que tienen que ver con características y atributos de los estudiantes como aspiraciones, valores y expectativas de éxitos.

En los modelos iniciales, se analiza la posibilidad de abandonar o permanecer en los estudios universitarios a partir de teorías adaptativas de los estudiantes a las instituciones, según antecedentes familiares, características de los estudiantes, entre otras. No presentan propuestas contradictorias, sino que se nutren unos de otros y es posible analizarlos de formas diversas. Existe una variable común a todos ellos, la relacionada con la habilidad académica del estudiante previo a su ingreso universitario. Sin embargo, no se profundiza en las variables pedagógicas.

De todos ellos, el modelo de Tinto es el más referenciado y utilizado para explicar la deserción-retención. La Tabla 1 recopila las variables identificadas como determinantes de la deserción o retención del estudiante en cada uno de dichos modelos, sus principales autores y fechas de publicación.

Estos modelos se han analizado en varias revisiones (Donoso \& Schiefelbein, 2007; Fonseca \& García, 2016; Suárez-Montes \& Díaz-Subieta, 2015). También han servido de referencia para la aportación de nuevos modelos en contextos específicos, tal es el caso del modelo del equilibrio dinámico centrado en el estudiante de permanencia en la universidad chilena (Díaz, 2008). Este se considera una combinación del modelo psicológico e interaccionista, en el que la deserción-retención se produce según el nivel de motivación que logra el estudiante como resultado de su integración académica y social.

En los años 90, países como Argentina, Brasil, Colombia, Cuba, Chile, Costa Rica y México implementaron sistemas nacionales de evaluación y acreditación, incluyendo dentro de sus indicadores de calidad, la eficiencia académica y la retención de los estudiantes. Al evaluar estos indicadores con muy bajos resultados, se identificó la necesidad de continuar los estudios para entender y atender la problemática de la deserción. Se considera la etapa del 1970 al 2000 como la etapa de propuesta y consolidación de los modelos iniciales de deserción/retención estudiantil.

A partir del 2000 se han publicado estudios interinstitucionales para analizar la problemática utilizando variables personales, familiares e institucionales (Pascarella, Pierson, Wolniak, \& Terenzini, 2004). Se han creado proyectos internacionales para profundizar en el tema, uno ejecutado por el Instituto de Educación Superior para América Latina y el Caribe (IESALC) con el objetivo de dimensionar la magnitud de la deserción universitaria en la región, donde participaron 15 países y se utilizaron datos de tres carreras de diferentes áreas del conocimiento: Derecho, Medicina e Ingeniería Civil, durante el periodo 1999-2003 (IESALC/UNESCO, 2006). 
Tabla 1

Variables identificadas por modelos iniciales de deserción-retención estudiantil. Fuente: Elaborada por los autores

\begin{tabular}{|c|c|c|c|c|c|}
\hline \multirow[t]{2}{*}{ Enfoque } & \multirow{2}{*}{$\begin{array}{l}\text { Modelo } \\
\text { Autor (fecha) y } \\
\text { Teoría que } \\
\text { utiliza }\end{array}$} & \multicolumn{4}{|c|}{ Variables } \\
\hline & & Familiares & Personales & Académicas & Institucionales \\
\hline Sociológico & $\begin{array}{l}\text { Spady (1970) } \\
\text { Teoría del } \\
\text { suicidio }\end{array}$ & $\begin{array}{l}\text { Influencias y } \\
\text { demandas }\end{array}$ & $\begin{array}{l}\text { Integración social } \\
\text { satisfacción, } \\
\text { potencial }\end{array}$ & Rendimiento & \\
\hline \multirow[t]{3}{*}{$\begin{array}{l}\text { Interaccionista } \\
\text { Organizacional }\end{array}$} & $\begin{array}{l}\text { Tinto (1975) } \\
\text { Teoría del } \\
\text { intercambio }\end{array}$ & $\begin{array}{c}\text { Situación } \\
\text { socioeconómica, } \\
\text { cultural y moral }\end{array}$ & $\begin{array}{c}\text { Habilidades y } \\
\text { capacidades } \\
\text { previas. Metas. } \\
\text { Persistencia }\end{array}$ & Rendimiento & $\begin{array}{l}\text { Interacción } \\
\text { académica y } \\
\text { social }\end{array}$ \\
\hline & $\begin{array}{l}\text { Bean (1980) } \\
\text { Modelo de la } \\
\text { productividad }\end{array}$ & $\begin{array}{c}\text { Condiciones } \\
\text { socioeconómicas }\end{array}$ & $\begin{array}{c}\text { Metas, } \\
\text { interacción. } \\
\text { Características: } \\
\text { género, edad, } \\
\text { lugar de } \\
\text { residencia... }\end{array}$ & Desempeño & $\begin{array}{l}\text { Adaptación y } \\
\text { compromiso }\end{array}$ \\
\hline & $\begin{array}{l}\text { Pascarella \& } \\
\text { Terenzini (1980) } \\
\text { Calidad del } \\
\text { esfuerzo }\end{array}$ & & $\begin{array}{l}\text { Antecedentes } \\
\text { académicos, } \\
\text { aptitud, } \\
\text { aspiración, } \\
\text { calidad del } \\
\text { esfuerzo }\end{array}$ & $\begin{array}{l}\text { Aprendizaje y } \\
\text { desarrollo } \\
\text { cognitivo }\end{array}$ & $\begin{array}{c}\text { Características } \\
\text { organizacionales } \\
\text { y estructurales. } \\
\text { Interacciones }\end{array}$ \\
\hline Psicológico & $\begin{array}{l}\text { (Ethington, 1990) } \\
\text { Conductas de } \\
\text { logro }\end{array}$ & $\begin{array}{l}\text { Antecedentes, } \\
\text { estímulo y apoyo }\end{array}$ & $\begin{array}{c}\text { Rendimiento } \\
\text { académico previo. } \\
\text { Aspiraciones y } \\
\text { valores. } \\
\text { Autoconcepto, } \\
\text { percepción de las } \\
\text { dificultades y } \\
\text { expectativas de } \\
\text { éxito. }\end{array}$ & & \\
\hline Económico & $\begin{array}{l}\text { (Cabrera, Nora, } \\
\text { \& Castañeda, } \\
\text { 1992) Enfoque } \\
\text { costo-beneficio }\end{array}$ & $\begin{array}{c}\text { Situación } \\
\text { socioeconómica }\end{array}$ & $\begin{array}{c}\text { Habilidad } \\
\text { académica previa, } \\
\text { costo de los } \\
\text { estudios, } \\
\text { beneficios } \\
\text { económicos }\end{array}$ & Desempeño & Apoyo financiero \\
\hline
\end{tabular}

Otro financiado por la Comunidad Europea, el proyecto Gestión Universitaria Integral del Abandono (ALFA GUIA, 2011) donde participaron 21 Instituciones de ES de Europa y Latinoamérica que proporciona un repositorio de 120 estudios explicativos de las causas e implicaciones de la deserción en diferentes instituciones, carreras y países; así como propuestas que contribuyan a elevar la persistencia y retención según el contexto. También financia anualmente la Conferencia Latinoamericana sobre el Abandono de la Educación Superior (CLABES), con el objetivo de intercambiar sobre aspectos relacionados con la calidad general de la enseñanza y, en particular, con el abandono de los estudios. Hemos considerado la etapa del 2010-2015 como el auge de estudios interinstitucionales. 
A partir de 2015 se considera la etapa actual donde varias instituciones han incrementado sus esfuerzos en la investigación de las causas de esta problemática en sus contextos. Algunos identificaron factores económicos, institucionales y sociales (Arce, Crespo, y Míguez, 2015; Barragán y González, 2015), otros autores han identificado factores sociofamiliares como problemas de enfermedad personales o de familiares (González-Rámirez y Pedraza-Navarro, 2017). También se han identificado factores relacionados con la vocación, la adaptación social y académica, la dedicación y el uso de técnicas de estudio, entre otras (Bernardo et al., 2016; Bulgarelli-Bolaños, Rivera-Rodríguez, y Fallas-Vargas, 2017; Da Re y Clerici, 2017) y aún más reciente (De Conincka et al., 2019) identificó la relación que existe entre la satisfacción, el rendimiento académico y la posibilidad de abandonar.

En el contexto cubano, los indicadores de calidad relacionados con la deserción que se utilizan por el Ministerio de Educación Superior (MES) son la retención y la eficiencia académica. Entendida la retención como la proporción de estudiantes que continúan en el sistema educativo, ya sea porque promueven al año siguiente o repiten un año académico, respecto a los que ingresaron. Por otra parte la eficiencia académica, que puede ser terminal o vertical se interpreta como:

- Eficiencia académica terminal es la proporción de egresados de la cohorte del total que matriculó el primer año al comenzar la carrera.

- Eficiencia académica vertical es la proporción de estudiantes de los diferentes años académicos que promovieron en un curso escolar.

A partir del estudio realizado en Cuba se propuso un modelo explicativo integrador de la manifestación de la eficiencia académica que presenta factores exógenos y endógenos a las Instituciones de Educación Superior (IES). Como factores endógenos se identificaron aquellos relacionados directamente con el estudiante, tales como: desempeño académico precedente, económicos, motivacionales, personales y familiares, dedicación al estudio y capacidad cognoscitiva. Dentro de los factores endógenos se incluyeron los relativos a la institución y el proceso docente, tales como: políticas educacionales, planes de estudio, requisitos de admisión para ingresar a las universidades, papel del docente, calidad del proceso de enseñanza aprendizaje, clima y prestigio de las instituciones, condiciones e infraestructura de las IES y reglamentos y normativas de la organización (Hernández, Vargas, Almuiñas, y García, 2015).

Según informe de la RED Iberoamericana de Indicadores de Educación Superior, la población de estudiantes en el periodo de 2010-2016 creció un 3.5\% anual en países de la región, sin embargo, en Cuba disminuyó al establecer a partir de 2010 como requisito de ingreso, la obligatoriedad de aprobar exámenes de ingreso (García, 2018). Elemento a tener en cuenta dentro de las variables a estudiar, sobre todo el examen de ingreso de Matemática, por la incidencia que esta materia tiene para las ingenierías.

También se han realizado transformaciones en la atención educativa dirigidas a garantizar la permanencia de los estudiantes en la ES (Peña-Hernández, Martínez-Sánchez, \& Jesús-Calderíus, 2019). Sin embargo, cerca del $40 \%$ de los estudiantes que ingresan a la ES en los cursos regulares diurnos no logran culminar sus estudios (Horruitiner, 2009). A pesar de ello, son escasos los estudios para predecir la deserción y no se han encontrado estudios similares al que se propone, relacionados con carreras del perfil de Ingeniería Informática.

Otro financiado por la Comunidad Europea, el proyecto Gestión Universitaria Integral del Abandono (ALFA GUIA, 2011) donde participaron 21 Instituciones de ES de Europa y Latinoamérica que proporciona un repositorio de 120 estudios explicativos de las causas e implicaciones de la deserción en diferentes instituciones, carreras y países; así como propuestas que contribuyan a elevar la persistencia y retención según el contexto. También financia anualmente la Conferencia Latinoamericana sobre el Abandono de la Educación Superior (CLABES), con el objetivo de intercambiar sobre aspectos relacionados con la calidad general de la enseñanza y, en particular, con el abandono de los estudios. Hemos considerado la etapa del 2010-2015 como el auge de estudios interinstitucionales.

A partir de 2015 se considera la etapa actual donde varias instituciones han incrementado sus esfuerzos en la investigación de las causas de esta problemática en sus contextos. Algunos identificaron factores económicos, institucionales y sociales (Arce, Crespo, y Míguez, 2015; Barragán y González, 2015), otros autores han identificado factores sociofamiliares como problemas de enfermedad personales o de familiares (González-Rámirez y Pedraza-Navarro, 2017). También se han identificado factores relacionados con la vocación, la adaptación social y académica, la dedicación y el uso de técnicas de estudio, entre otras (Bernardo et al., 2016; Bulgarelli-Bolaños, Rivera-Rodríguez, y Fallas-Vargas, 2017; Da Re y Clerici, 2017) y 
aún más reciente (De Conincka et al., 2019) identificó la relación que existe entre la satisfacción, el rendimiento académico y la posibilidad de abandonar.

En el contexto cubano, los indicadores de calidad relacionados con la deserción que se utilizan por el Ministerio de Educación Superior (MES) son la retención y la eficiencia académica. Entendida la retención como la proporción de estudiantes que continúan en el sistema educativo, ya sea porque promueven al año siguiente o repiten un año académico, respecto a los que ingresaron. Por otra parte la eficiencia académica, que puede ser terminal o vertical se interpreta como:

- Eficiencia académica terminal es la proporción de egresados de la cohorte del total que matriculó el primer año al comenzar la carrera.

- Eficiencia académica vertical es la proporción de estudiantes de los diferentes años académicos que promovieron en un curso escolar.

A partir del estudio realizado en Cuba se propuso un modelo explicativo integrador de la manifestación de la eficiencia académica que presenta factores exógenos y endógenos a las Instituciones de Educación Superior (IES). Como factores endógenos se identificaron aquellos relacionados directamente con el estudiante, tales como: desempeño académico precedente, económicos, motivacionales, personales y familiares, dedicación al estudio y capacidad cognoscitiva. Dentro de los factores endógenos se incluyeron los relativos a la institución y el proceso docente, tales como: políticas educacionales, planes de estudio, requisitos de admisión para ingresar a las universidades, papel del docente, calidad del proceso de enseñanza aprendizaje, clima y prestigio de las instituciones, condiciones e infraestructura de las IES y reglamentos y normativas de la organización (Hernández, Vargas, Almuiñas, y García, 2015).

Según informe de la RED Iberoamericana de Indicadores de Educación Superior, la población de estudiantes en el periodo de 2010-2016 creció un 3.5\% anual en países de la región, sin embargo, en Cuba disminuyó al establecer a partir de 2010 como requisito de ingreso, la obligatoriedad de aprobar exámenes de ingreso (García, 2018). Elemento a tener en cuenta dentro de las variables a estudiar, sobre todo el examen de ingreso de Matemática, por la incidencia que esta materia tiene para las ingenierías.

También se han realizado transformaciones en la atención educativa dirigidas a garantizar la permanencia de los estudiantes en la ES (Peña-Hernández, Martínez-Sánchez, \& Jesús-Calderíus, 2019). Sin embargo, cerca del $40 \%$ de los estudiantes que ingresan a la ES en los cursos regulares diurnos no logran culminar sus estudios (Horruitiner, 2009). A pesar de ello, son escasos los estudios para predecir la deserción y no se han encontrado estudios similares al que se propone, relacionados con carreras del perfil de Ingeniería Informática.

\section{Estudios de deserción en carreras de Ingeniería}

Para realizar un estudio del estado de la cuestión, se ha realizado una búsqueda formal y sistemática en la colección principal de Web of Science (WoS). Se escogió esta base por contemplar fuentes de alto prestigio y ser el recurso empleado de facto en la literatura para analizar el estado de un ámbito de estudio o incluso inferir los tópicos candentes de investigación en un área (Úbeda-Sánchez, Fernández-Cano, \& Callejas, 2019).

La búsqueda en WoS Core Collection se realizó el día 30/04/2019, con lo que contamos con un intervalo temporal amplio, estable y acotado desde las primeras publicaciones indexadas en WoS hasta el día de la búsqueda. El criterio de la búsqueda en el campo "topic"fue: drop-out AND university de forma que se recogiesen resultados que contuviesen ambos términos en título, resumen o palabras clave. Esta búsqueda arrojó 846 resultados.

Refinamos el criterio "dominios de aplicación" considerando únicamente SOCIAL SCIENCES (para cubrir las publicaciones de educación y pedagogía) y SCIENCE AND TECHNOLOGY (para considerar publicaciones relacionadas con los estudios de informática). A pesar de estas restricciones, dentro del ámbito de ciencia y tecnología había numerosas investigaciones donde drop-out se refiere a la adherencia a medicaciones o estudios. Para excluirlos, se refinó también el apartado "Áreas de investigación" excluyendo aquellas relacionadas con servicios de ciencias de la salud (health care science services), psiquiatría (psychiatry), farmacología (pharmacology pharmacy), pediatría (pediatrics) y en general todos los relacionados con la medicina.

El resultado final fueron 522 trabajos indexados, que pueden consultarse en (SelecciónWoS, 2019). Todos los artículos recabados fueron exportados a Mendely para su posterior lectura y procesado. 
En cuanto a los países de los investigadores que han realizado dichas publicaciones, destacan Estados Unidos (80 artículos), España (69 artículos), Inglaterra (53 artículos), Alemania (36 artículos) e Italia (23 artículos). En cuanto a los países latinoamericanos, sólo hay 3 entre los 20 primeros puestos (Brasil en el puesto 9 con 16 trabajos, México en el puesto 16 con 10 y Argentina en el 17 con 6). Hay una única contribución como resultado de esta búsqueda donde participa un autor cubano, con lo que Cuba figura en el puesto 50 y ésta no se refiere a la deserción en universidades cubanas (Estrada et al., 2011).

Los resultados anteriores muestran la pertinencia de un estudio de deserción estudiantil universitaria en el contexto cubano en un área de investigación en ascenso y de gran interés para la comunidad científica internacional.

Se encontraron solo 43 estudios enfocados en la deserción en carreras técnicas. Se eliminaron de ellas las que están relacionadas con cursos en la modalidad a distancia y tres que no analizan factores de deserción, sino que hacen propuestas pedagógicas para evitarlas; quedando finalmente 24 artículos a analizar relacionados con el estudio de causas de deserción en Informática. Dichos artículos se clasificaron según las variables estudiadas (ver Tabla 1) en sociológicos, psicológicos, interaccionista, organizacional o la combinación de ellos. En la Tabla 2 se resumen estos estudios seleccionando los siguientes indicadores: tipo de estudio, país (Autores, año).

Como se puede apreciar la problemática afecta a diferentes países. La mayoría de los estudios se clasifican como psicológicos, sociológicos o la combinación de estos por las variables que analizan, la metodología que predomina es la cuantitativa y solo tres utilizan mixta.

(Paimin, et al., 2017) estudian los factores psicológicos que influyen en la capacidad de los estudiantes para permanecer y esforzarse en los programas de Ingeniería en una Universidad de Malasia y Australia. (Soistak et al., 2018) obtienen que el 61\% de los estudiantes que abandonaron entre 2013 y 2014 en la Universidad Tecnológica Federal de Paraná, lo hicieron por problemas académicos. En Alemania, las matemáticas, la informática, la física, ciencias de la tierra y la química se caracterizan por un alto grado de deserción de más del $30 \%$, así como de $34 \%$ y $33 \%$ en los campos de ingeniería mecánica e ingeniería eléctrica, respectivamente (Heublein y Wolter, 2011, p 222).

Por otra parte, la discusión sobre diferencias de género en el abandono en Informática es frecuente, (Miliszewska et al., 2006) reporta que un 40\% de mujeres abandonan la carrera de Informática. También se determinan como variables predictivas la preparación previa en Matemática, relacionada con los puntajes en las pruebas de acceso y el rendimiento académico en primer año, sobre todo en las asignaturas de Matemática e Informática introductoria (Canedo et al., 2018; Heublein y Wolter, 2011; Kori, et al., 2018; Niitsoo et al., 2014).

Se analizan variables que no se valoran en el estudios anteriores como son: las competencias teóricas y prácticas con computadoras (Araque et al., 2009; Canedo et al., 2018; Xenos et al., 2002; Zehetmeier et al., 2015), el desarrollo del pensamiento lógico y analítico (Salazar-Fernandez et al., 2019; Zehetmeier et al., 2015) y la integración profesional (Kori et al., 2018). A pesar que se analizan variables socioeconómicas ninguno de ellos tiene un enfoque economicista.

En general los estudios revisados sobre deserción estudiantil en carreras de Ingeniería y en particular Informática, estudian variables ya identificadas en los modelos iniciales de deserción. Sin embargo, introducen variables pedagógicas como es el proceso de enseñanza aprendizaje de la Matemática (Alzen et al., 2018) la motivación por el estudio de dichas carreras (Meyer \& Fang, 2019; Paimin et al., 2017) los estilos de aprendizaje y métodos de enseñanza (Van Den Broeck et al., 2017).

En resumen, hemos detectado diversos factores previos y posteriores al ingreso de los estudiantes en las instituciones de ES. Se puede concluir que aún son pocas las investigaciones sobre la deserción en estas carreras. 
Tabla 2

Selección de artículos sobre factores de deserción en carreras de perfil ingeniería informática organizadas por tipo de estudio. Fuente: elaboración propia

\begin{tabular}{|c|c|}
\hline Tipos de estudios & País (Autores, año) \\
\hline Sociológico & $\begin{array}{ll}\text { - } & \text { Chile (Salazar-Fernandez, Sepúlveda, y Munoz-Gama, 2019) } \\
\text { - } & \text { EEUU (Meyer y Fang, 2019) } \\
\text { - } & \text { Brasil (Soistak et al., 2018) } \\
\text { - } & \text { Estonia (Altin y Rantsus, 2015) }\end{array}$ \\
\hline Psicológico & $\begin{array}{ll}\text { - } & \text { Malasia y Australia (Paimin, Prpic, Hadgraft, y Alias, 2017) } \\
\text { - } & \text { Bélgica (Van Den Broeck et al., 2017) } \\
\text { - } & \text { Brasil (Portella, de Melo, y Teixeira, 2015) } \\
\text { - } & \text { Estonia (Niitsoo, Paales, Pedaste, Siiman, y Tõnisson, 2014) } \\
\text { - } & \text { Finlandia (Paivi, 2012) } \\
\text { - } & \text { Argentina (Maris y Difabio, 2009) } \\
\text { - } \quad \text { China (Yong-Min, Ai-bin, y Jun, 2009) } \\
\text { - } \quad \text { Grecia (Xenos, Pierrakeas, y Pintelas, 2002) }\end{array}$ \\
\hline Interaccionista & $\begin{array}{ll}\text { - } & \text { EEUU (Alzen, Langdon, y Otero, 2018) } \\
\text { - } & \text { Estonia (Kori, Pedaste, y Must, 2018) } \\
\text { - } & \text { Sudáfrica (Kuriakose, 2017) }\end{array}$ \\
\hline Organizacional & $\begin{array}{ll}\text { - } & \text { Brasil (Canedo, Santos, y Leite, 2018) } \\
\text { - } & \text { España (Lacave, Molina, y Cruz-Lemus, 2018) } \\
\text { - } & \text { Australia (Miliszewska, Barker, Henderson, y Sztendur, 2006) }\end{array}$ \\
\hline $\begin{array}{l}\text { Sociológico y } \\
\text { psicológico }\end{array}$ & $\begin{array}{ll}\text { - } & \text { Alemania (Zehetmeier, Axel, Bruggemann-Klein, Thurner, y Schlierkamp, 2015) } \\
\text { - } & \text { Sudáfrica (Ahmed, Kloot, y Collier-Reed, 2015) } \\
\text { - } & \text { Alemania y Austria (Elster, 2014) } \\
\text { - } & \text { Alemania (Heublein y Wolter, 2011) } \\
\text { - } & \text { España (Araque, Roldán, y Salguero, 2009) }\end{array}$ \\
\hline $\begin{array}{l}\text { Interaccionista y } \\
\text { organizacional }\end{array}$ & - $\quad$ Argentina (Graffigna et al., 2014) \\
\hline
\end{tabular}

\section{Materiales y método}

En base a los factores detectados en la revisión realizada en la sección anterior y a los datos de los estudiantes de nuevo ingreso a carreras de perfil Ingeniería Informática en Cuba, se describe a continuación, el estudio realizado con una muestra de estudiantes pertenecientes a todas las provincias del país de la cohorte 2013-2014 para identificar factores predictivos de deserción estudiantil.

Se plantean las siguientes hipótesis, que aparecen resumidas en la Figura 1.

- Hipótesis $1(\mathrm{H} 1)$. El género, la provincia de procedencia, la opción en que solicitó la carrera, la nota del examen de ingreso de Matemática y la fuente de ingreso inciden en la deserción estudiantil en carreras de Ingeniería Informática.

- Hipótesis $2(\mathrm{H} 2)$. La nota del examen de ingreso de Matemática incide directamente en el rendimiento académico en Matemática y Programación.

- Hipótesis $3(\mathrm{H} 3)$. El rendimiento académico en Matemática y Programación incide inversamente en la deserción estudiantil en carreras de Ingeniería Informática.

- Hipótesis $4(\mathrm{H} 4)$. La repitencia del primer año académico incide directamente en la deserción estudiantil en carreras de Ingeniería Informática. 


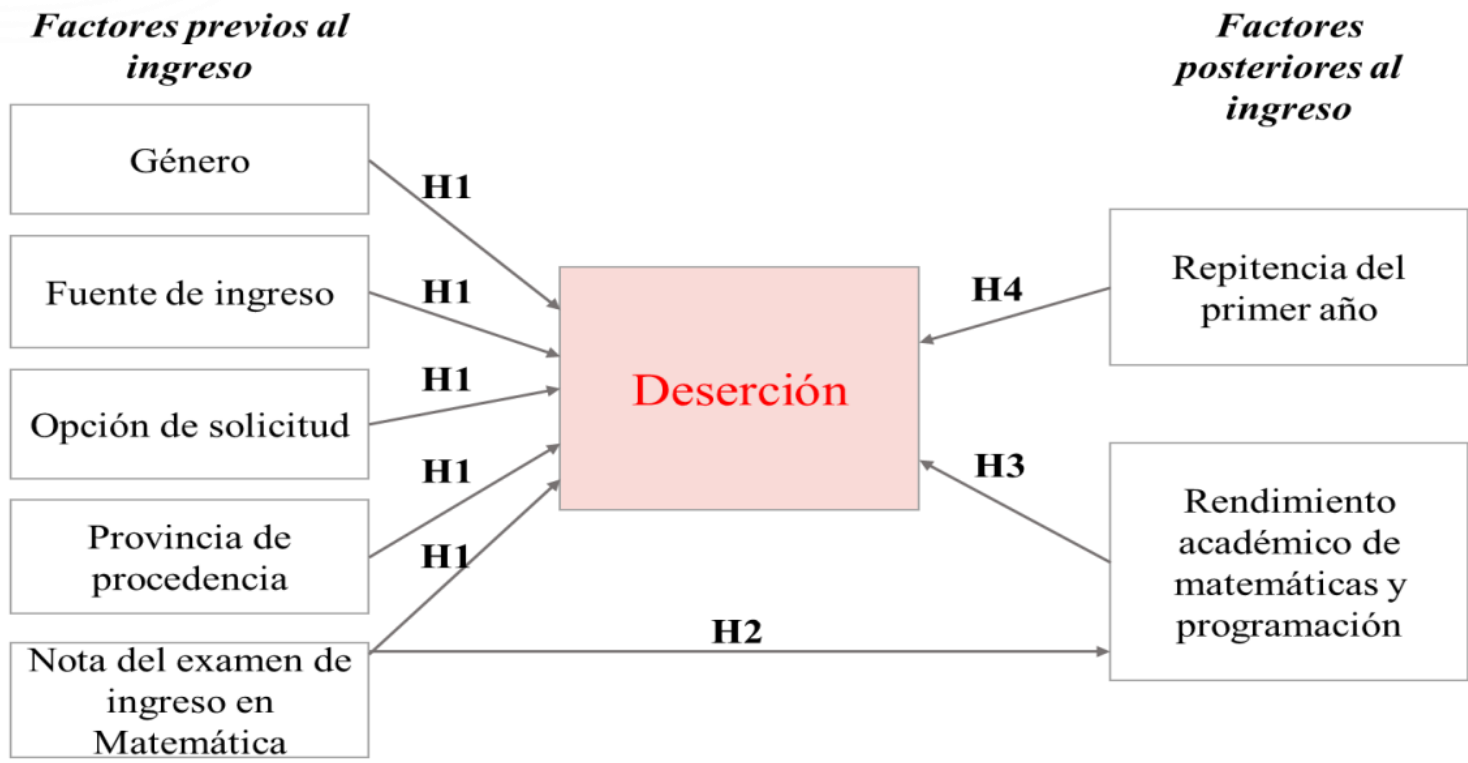

Figura 1. Hipótesis planteadas para la investigación

Luego de identificar mediante un estudio teórico los factores expuestos en las secciones 2 y 3 , se seleccionaron las variables para realizar un estudio cuantitativo. De entre las analizadas en estudios precedentes, se tuvo acceso en el Sistema de Gestión Universitaria a las que aparecen en las hipótesis planteadas. Aunque no se pudo acceder a la variable socioeconómica, no tiene tanto impacto como en otros contextos teniendo en cuenta que en Cuba la Educación es gratuita, incluyendo los materiales de estudio, residencia estudiantil, alimentación. Se garantizan además por el estado, ayudas económicas o préstamos estudiantiles a los estudiantes que lo soliciten y se asigna un estipendio mensual a todos los estudiantes universitarios.

En primer lugar, se ha realizado un análisis descriptivo de las variables en estudio para inferir características de las mismas en la población; en segundo, se realizó un análisis de la correlación existente entre las variables en estudio (Hernández-Sampieri, Fernández-Collado, y Baptista-Lucio, 2014).

En tercer lugar, se utilizó la regresión logística con el objetivo de obtener el modelo matemático que sirva para calcular la probabilidad de que un estudiante cause baja de la institución y con ello predecir si un estudiante está en riesgo de deserción (Berlanga-Silvente \& Vilà-Baños, 2014). De este tercer análisis solo se dará a conocer las variables que resultaron predictivas por restricciones de espacio.

Por último, se realizó un análisis discriminante para identificar las variables cuantitativas que discriminan o diferencian los estudiantes en los que desertan de la carrera, repiten el primer año académico o promueven, así como para crear una función discriminante capaz de clasificar de forma más precisa a los estudiantes a partir de conocer los datos de las variables que discriminan (Austin \& Steyerberg, 2015; Mendoza \& Acosta, 2013). El análisis se realizó utilizando el Paquete Estadístico para las Ciencias Sociales (SPSS versión 22).

\subsection{Población y muestra}

La población seleccionada para el estudio está compuesta por los 1022 estudiantes de todas las provincias cubanas, que matricularon el primer año en carreras de perfil Ingeniería Informática en el curso académico 2013-2014. La muestra está formada por 485 estudiantes de nuevo ingreso de todas las provincias, lo que representa el $48 \%$ de los estudiantes matriculados en primer año ese curso en todo el país en carreras de dicho perfil, se seleccionó una muestra representativa de todas las provincias, teniendo en cuenta los datos a analizar y se excluyeron todos aquellos estudiantes que carecían de, al menos, uno de los datos en estudio que se describen en la siguiente sección. De ellos 178 (37\%) son mujeres y 307 (63\%) hombres.

La representatividad de la muestra en relación a la población por provincias se presenta en la Figura 2, la muestra oscila entre un $40 \%$ y un $50 \%$ de la población de cada provincia, después de desechar los casos que no se cuenta con todos sus datos. 


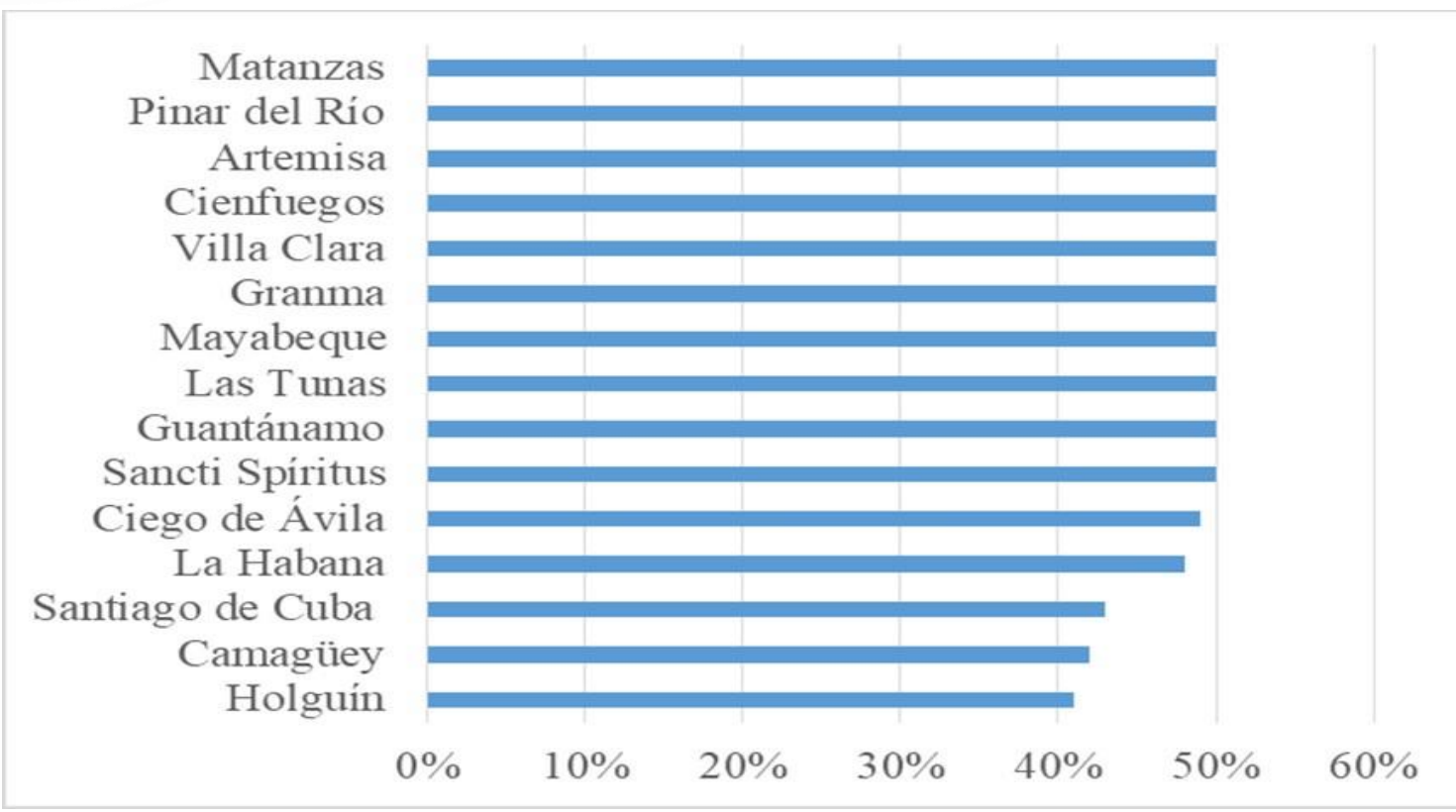

Figura 2. Porcentaje representativo de la muestra respecto a la población por provincias

\subsection{Variables}

Las variables correspondientes a los factores identificados se obtuvieron a partir de datos de los estudiantes almacenados en diversas bases de datos institucionales, algunos se recogieron en entrevistas a los estudiantes en el momento del ingreso y otros en el transcurso del curso escolar. Se describen a continuación cada una de las variables seleccionadas, que se presentan resumidas en la Tabla 3.

\section{Tabla 3}

Descripción de las variables. Fuente: Elaborada por los autores

\begin{tabular}{|c|c|c|}
\hline Variable & Tipo & Valores \\
\hline Deserción & Nominal & (0) No, (1) Sí \\
\hline $\begin{array}{l}\text { Deserción: Situación escolar en el curso } \\
15-16\end{array}$ & Nominal & (1)Baja, (2) Repite y (3) Promueve \\
\hline Género & Nominal & Masculino (0) Femenino (1) \\
\hline Provincia de procedencia & Nominal & $\begin{array}{l}\text { (1) Las Tunas, (2) Guantánamo, (3) Ciego de Ávila, (4) } \\
\text { Sancti Spíritus, (5) Santiago de Cuba, (6) Mayabeque, } \\
\text { (7) Artemisa, (8) Villa Clara, (9) Holguín, (10) } \\
\text { Cienfuegos, (11) Pinar del Río, (12) Granma, (13) } \\
\text { Camagüey, (14) Matanzas, (15) La Habana }\end{array}$ \\
\hline Rango de opción en que solicitó la carrera & Intervalo & (0) entre $4^{\mathrm{a}}$. y $10^{\mathrm{a}}$, ,(1) $2^{2}$. $.3^{\mathrm{a}}$. ,(2) $1^{\mathrm{a}}$. opción \\
\hline Fuente de ingreso & Nominal & (1) otras, (2) TMI, (3) IPU, (4) IPVCE \\
\hline $\begin{array}{l}\text { Rango de nota en el examen de ingreso } \\
\text { de Matemática (EIM) }\end{array}$ & Intervalo & $\begin{array}{l}\text { (1) de } 60 \text { a } 69, \text { (2) de } 70 \text { a } 79, \text { (3) de } 80 \text { a } 89, \text { (4) de } 90 \\
\text { a } 100\end{array}$ \\
\hline $\begin{array}{l}\text { Rendimiento académico en Matemática y } \\
\text { Programación (RAMP) }\end{array}$ & Nominal & (0) Bajo, (1) Medio, (2) Alto \\
\hline Repitencia del primer año académico & Nominal & (0) No, (1) Sí \\
\hline
\end{tabular}




\subsubsection{Variable dependiente: Deserción estudiantil en carreras de perfil Ingeniería Informática}

En Cuba, el MES define el término considerado para esta investigación como deserción con el término de baja. El Reglamento de organización docente de la ES plantea que: "Se entiende por baja la suspensión temporal o definitiva de la condición de estudiante universitario" (MES, 2017, p.15) considerando además diferentes tipos de baja: por insuficiencia docente, por sanción disciplinaria, voluntaria, por deserción, por pérdida de requisitos, por inasistencia y definitiva. A partir de ahora cuando se hable de "deserción" se entiende que es baja por cualquier causa del Reglamento.

El estudiante que no causa baja, en dependencia de sus resultados académicos, puede promover el año inmediato superior o repetir el año académico que cursa, esta última opción solo en una oportunidad. Por tanto, cada estudiante presenta una situación escolar al concluir cada curso escolar que se nombran: baja, repite o promueve, esta clasificación se utilizará en el análisis discriminante.

La variable dependiente deserción para el análisis de correlación se considera binaria: si causa baja (deserción para esta investigación) y si no causa baja (permanencia) ya sea el que repite el año académico o el que promueve al segundo año.

\subsubsection{Variable independiente: nota del examen de ingreso en Matemática}

Los únicos requisitos para ingresar o acceder a las universidades en Cuba son culminar la enseñanza preuniversitaria (enseñanza entre la secundaria y la universidad en Cuba) y aprobar las tres pruebas de ingreso propuestas por el MES. Dichas pruebas son iguales para todos los estudiantes del país y se realizan de forma simultánea mediante tres exámenes: Matemática, Español e Historia de Cuba. Las calificaciones de dichas pruebas utilizan el baremo 0-100 puntos.

Para la carrera en estudio se considera de gran valor la nota obtenida por el estudiante en Matemática, un estudiante puede ingresar a la ES si su calificación es igual o superior a 60 . En este estudio la variable se agrupó en cuatro rangos: de 60 a 69 puntos, de 70 a 79, de 80 a 89 y de 90 a 100.

\subsubsection{Variables independientes: género, provincia de procedencia, fuente de ingreso y opción en que} solicitó la carrera

En los estudios de deserción estudiantil siempre se utiliza la variable género o sexo. En carreras de ciencias técnicas, generalmente se ha comprobado que los hombres permanecen más que las mujeres (SuárezMontes y Díaz-Subieta, 2015). También se estudian variables demográficas como la procedencia de los estudiantes, ya sea el lugar donde viven o los estudios precedentes (Bonaldo \& Nobre, 2016).

Los estudiantes, previo al ingreso a la ES, solicitan 10 opciones de carreras en orden de preferencia; luego de realizar los exámenes de ingreso a la ES el estudiante obtiene la carrera universitaria a partir de un escalafón provincial. El otorgamiento definitivo se realiza a partir de ordenar a los estudiantes de forma ascendente en dicho escalafón, por lo que según estos resultados, en ocasiones se le asigna la carrera menos deseada. De ahí la importancia de estudiar qué incidencia tiene la opción en que se solicitó la carrera en la decisión del estudiante de permanecer o abandonarla durante o al culminar su primer año de estudios.

- Género: Hombres y mujeres.

- Provincia de procedencia: Las 15 provincias del país descritas en la Tabla 3.

- La fuente de ingreso: Término utilizado en el contexto cubano para indicar la procedencia educativa de los estudiantes que acceden a la ES (MES, 2014). Los estudiantes provienen de diferentes fuentes de ingreso, la de mayor ingreso son los Institutos Preuniversitarios (IPU), le siguen los Institutos Preuniversitarios Vocacionales de Ciencias Exactas (IPVCE) donde se fortalece la preparación en Matemática, Física y Química. También ingresan estudiantes de Técnico Medio en Informática (TMI) con formación profesional, así como otras fuentes de ingreso que aportan menor cantidad de estudiantes.

- Opción en que solicitó la carrera: Se dividió en tres rangos: (i) primera opción, (ii) segunda o tercera opción y (iii) de la cuarta a la décima opción. 


\subsubsection{Variable independiente: rendimiento académico en Matemática y Programación}

El rendimiento académico en una asignatura universitaria en Cuba se evalúa empleando las categorías siguientes (MES, 2007): excelente (5), bien (4), regular (3) y mal (2). Cada categoría establecida expresa el grado de calidad alcanzado por el estudiante en el cumplimiento de los objetivos de las asignaturas. Las calificaciones de 5,4 y 3 expresan diferentes grados de dominio de los objetivos que tienen los estudiantes y resulta aprobado en la asignatura; la calificación de 2 expresa que el estudiante no domina los objetivos al nivel requerido. Se analizará qué incidencia tiene el rendimiento académico en las asignaturas de Matemática y Programación en la deserción estudiantil.

- El rendimiento académico en Matemática y Programación (RAMP): Los estudiantes reciben en el primer año de la carrera cinco asignaturas básicas de la disciplina Matemática: Álgebra Lineal, Matemática I y II, Matemática Discreta I y II en ambos semestres; así como tres asignaturas de la especialidad: Introducción a las Ciencias Informáticas, Introducción a la Programación y Programación I. EI RAMP se calcula como el porcentaje de asignaturas aprobadas de las ocho que recibió en el primer año.

Para el análisis de correlación y la RL el RAMP se clasifica en alto, medio y bajo. Se consideró alto si tiene todas las asignaturas aprobadas, medio si tiene 1 o 2 asignaturas suspensas y bajo si suspende 3 o más asignaturas.

\subsubsection{Variable independiente: repitencia del primer año académico}

En la ES cubana un estudiante que desaprueba dos asignaturas en un mismo semestre o más de dos asignaturas en el curso, podrá valorarse la posibilidad de repetir el año académico, se tendrá en cuenta que sus resultados docentes no estén vinculados a una mala actitud ante el estudio. Se considera oportuno analizar si esta variable incide en la deserción.

- Repitencia del primer año: Hemos analizado la relación que existe entre los estudiantes de la muestra que repitieron el primer año en el curso 2014-2015 con la deserción, o sea, su situación escolar al concluir el año de repitencia.

\section{Presentación y discusión de resultados}

La distribución de la muestra por géneros y situación escolar en el curso 2013-2014, o sea, estudiantes que causaron baja, repiten el primer año o promueven al segundo, se aprecia en la Figura 3. De la muestra total $N=485$ de estudiantes, el 62\% (299) promueve, 18\% (89) repite el primer año y $20 \%$ (97) causa baja. En los tres casos el sexo masculino supera al femenino.

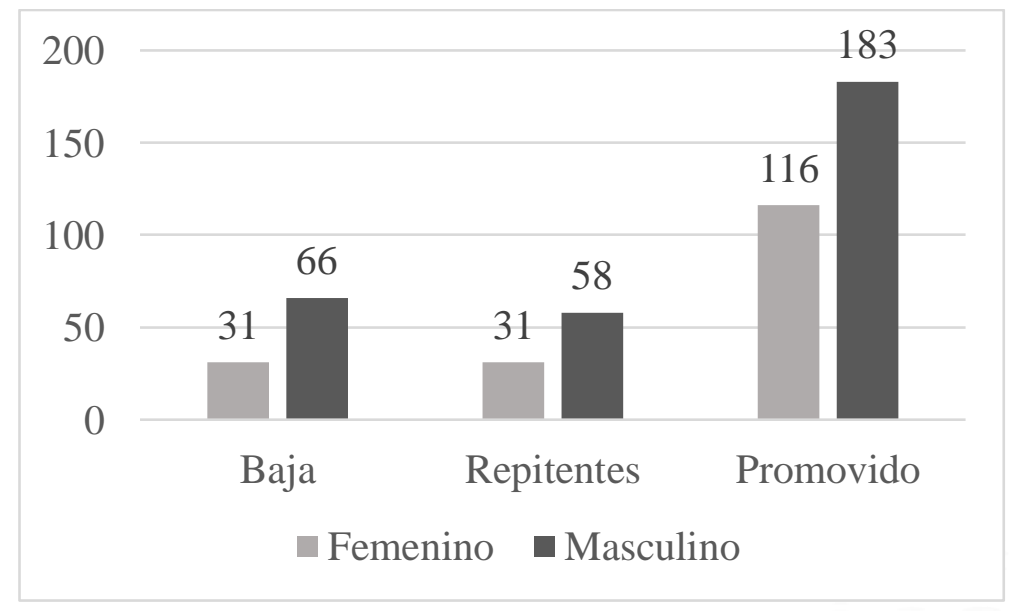

Figura 3. Situación escolar de los estudiantes en el curso 2013-2014 por género 
El ingreso a la ES en Cuba se planifica por provincias según la demanda de empleos al concluir el periodo lectivo de cinco años, la provincia que más estudiantes ingresó fue La Habana y aparece en la Tabla 4 la distribución de la muestra $N=485$ estudiantes por todas las provincias del país, que matricularon a carreras de perfil Ingeniería Informática en el curso escolar 2013-2014.

\section{Tabla 4}

Distribución de estudiantes por provincias, porcentaje respecto a la muestra

\begin{tabular}{lrr}
\hline Provincia & Frecuencia & Porcentaje \\
\hline Las Tunas & 13 & $2,7 \%$ \\
Guantánamo & 14 & $2,9 \%$ \\
Ciego de Ávila & 16 & $3,3 \%$ \\
Sancti Spiritus & 16 & $3,3 \%$ \\
Santiago de Cuba & 20 & $4,1 \%$ \\
Mayabeque & 21 & $4,3 \%$ \\
Artemisa & 22 & $4,5 \%$ \\
Villa Clara & 27 & $5,6 \%$ \\
Holguin & 27 & $5,6 \%$ \\
Cienfuegos & 32 & $6,6 \%$ \\
Pinar del Rio & 32 & $6,6 \%$ \\
Granma & 34 & $7,0 \%$ \\
Camagüey & 38 & $7,8 \%$ \\
Matanzas & 46 & $9,5 \%$ \\
La Habana & 127 & $26,2 \%$ \\
Total & $\mathbf{4 8 5}$ & $\mathbf{1 0 0 , 0} \%$ \\
\hline
\end{tabular}

La deserción estudiantil se determinó a partir de la Situación Escolar (SE) que presentaron los estudiantes al inicio del curso escolar siguiente, o sea, el curso 2014-2015, ellas son: baja (deserción o abandono), repitencia del primer año o promueve.

Primeramente, para analizar si existe relación entre las variables independientes y la deserción, se realizó la prueba Chi cuadrado con $N=485$ pues todas las variables se expresan de forma categórica. Del resultado en la Tabla 5 se concluye que las variables provincia de procedencia, rango de nota del EIM y RAMP se relacionan con la variable deserción estudiantil.

\section{Tabla 5}

Resultados de la prueba Chi-cuadrado entre las Variables Independientes y la Deserción estudiantil

\begin{tabular}{llll}
\hline & $\begin{array}{l}\text { Valor Chi- } \\
\text { cuadrado de } \\
\text { Pearson }\end{array}$ & gl & $\begin{array}{l}\text { Sig. asintótica } \\
\text { (2 caras) }\end{array}$ \\
\hline Variables & 1.174 & 1 & .279 \\
Género & 33.277 & 14 & .003 \\
Provincia de procedencia & 5.855 & 3 & .119 \\
$\begin{array}{l}\text { Fuente de ingreso } \\
\text { Rango de opción en que solicitó la carrera }\end{array}$ & 4.382 & 2 & .112 \\
$\begin{array}{l}\text { Rango de nota del examen de ingreso en } \\
\text { Matemática }\end{array}$ & 21.784 & 3 & .000 \\
$\begin{array}{l}\text { Rendimiento académico en Matemática y } \\
\text { Programación }\end{array}$ & 182.994 & 2 & .001 \\
Repitencia del primer año & 1.287 & 1 & .257 \\
\hline
\end{tabular}


A continuación, se muestra el resultado de los coeficientes de correlación por hipótesis. Se utilizó Rho de Spearman pues se realiza un análisis no paramétrico con variables ordinales o de rangos, que se muestra en la Tabla 6.

\section{Tabla 6}

Resultados de correlación de Spearman entre las variables Género, Provincia de procedencia, Opción en que solicitó la carrera, Fuente de ingreso y Nota del EIM con la Deserción estudiantil en carreras de Ingeniería Informática.

\begin{tabular}{lll}
\hline & & $\begin{array}{c}\text { Rho de Spearman } \\
\text { con Deserción }\end{array}$ \\
\hline Género & Coeficiente de correlación & -.049 \\
& Sig. (bilateral) & .280 \\
Provincia de procedencia & Coeficiente de correlación & $.191^{* *}$ \\
& Sig. (bilateral) & .000 \\
Fuente de ingreso & Coeficiente de correlación & .041 \\
& Sig. (bilateral) & .368 \\
Rango de opción en que solicitó & Coeficiente de correlación & $-.094^{*}$ \\
la carrera & Sig. (bilateral) & .039 \\
Rango de nota del examen de & Coeficiente de correlación & $.228^{* *}$ \\
ingreso en Matemática & Sig. (bilateral) & .000 \\
\hline
\end{tabular}

** La correlación es significativa en el nivel de .01 (dos colas)

* La correlación es significativa en el nivel de .05 (dos colas)

De esta se infiere para la $\mathrm{H} 1$ que:

- Género: Ingresan más hombres que mujeres y en los dos casos deserción o permanencia, el sexo masculino supera al femenino. No es significativa la relación.

- Provincia de procedencia: De 15 provincias en total se aprecia que 5 de las 9 provincias que menos estudiantes ingresaron tuvieron el menor porciento de promovidos, mientras 4 de las 6 provincias que más estudiantes ingresaron tienen el mayor porciento de promovidos. Se obtuvo una correlación significativa con un nivel de significación $\mathrm{p}<.01$.

- Fuente de ingreso: No se obtiene una relación significativa. Se puede apreciar que el mayor por ciento de los estudiantes que proceden de los TMI e IPVCE promueven, con un $92 \%$ y $87 \%$, respectivamente. Asimismo, de la mayor fuente de ingreso que son los IPU, permanece el $79 \%(297)$ y causan baja 81 , que representan el $84 \%$ de todos los estudiantes que causan baja.

- Opción en que solicitó la carrera: De $N=485$ los tres rangos de opción se distribuyen de la siguiente manera: (i) 170 estudiantes (35\%) seleccionaron en $1^{\text {a }}$ opción, (ii) 138 seleccionaron la $2^{\underline{a}} \circ 3^{\text {a }}(28 \%)$ y (iii) 177 estudiantes (37\%) solicitaron entre $4^{\underline{a}}$ y $10^{\underline{a}}$ opción. De los 97 estudiantes que causan baja, el $42 \%$ solicitó en primera opción, y de los 388 que permanecieron el $39 \%$ solicitó entre la $4^{\underline{a}}$ y $10^{\text {a }}$ opción. Existe una pequeña relación inversa entre esta variable y la deserción escolar pues el valor de significación $\mathrm{p}<.05$.

- Nota en el EIM: El estudiante ingresa a la ES si obtiene más de 60 puntos de 100 en los tres exámenes de ingreso, explicado en el apartado 4.2.3, la que se distribuye en la muestra como aparece en Figura 4. El 54\% (52) de los estudiantes que causan baja, obtuvieron notas en el examen de ingreso entre 60 y 69. Existe correlación significativa con $p<.01$. 


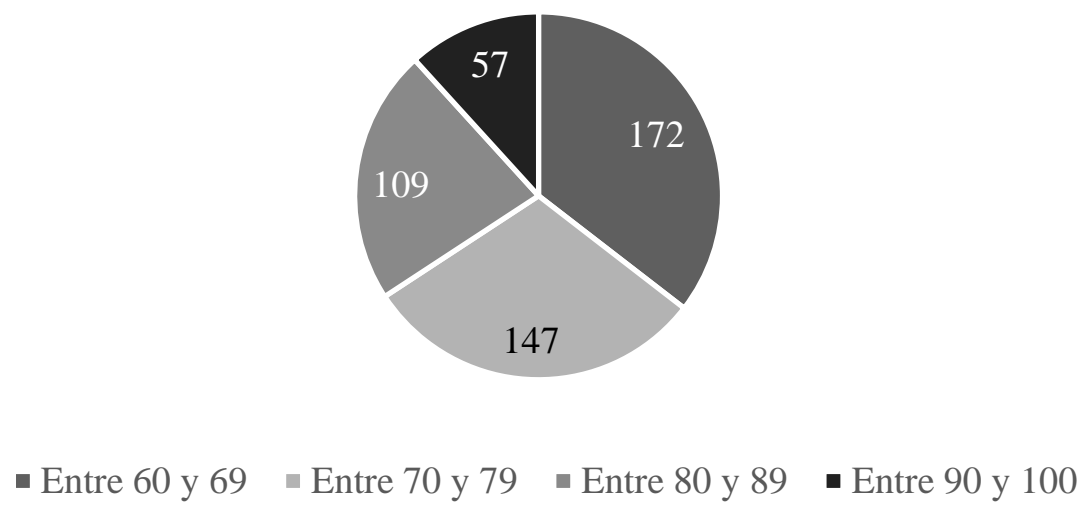

Figura 4. Cantidad de estudiantes de la muestra $\mathrm{N}=485$ por rango de notas del examen de ingreso en Matemática

Para la $\mathrm{H} 1$ se puede apreciar que la provincia de procedencia, opción en que solicitó la carrera y la nota en el EIM inciden en la deserción, no inciden las variables género y fuente de ingreso, por lo que se acepta parcialmente $\mathrm{H} 1$.

Las variables de la $\mathrm{H} 2$ son el rango de nota del EIM y el RAMP. El RAMP se consideró alto, medio y bajo según se explica en la sección 4.2.4. El resultado de la correlación permite aceptar la hipótesis de investigación ya que $R_{s}(6)=.338^{* *}, \mathrm{p}<.01$.

Para entender la correlación se utilizó la tabulación cruzada y se puede apreciar en la Figura 5 que 172 estudiantes $(36 \%)$ de la matrícula inicial obtuvieron un rango de nota entre 60 y 69, incluso, 319 estudiantes $(66 \%)$ de la matrícula inicial, ingresaron con notas del EIM para acceder a la Universidad, por debajo de 80 puntos.

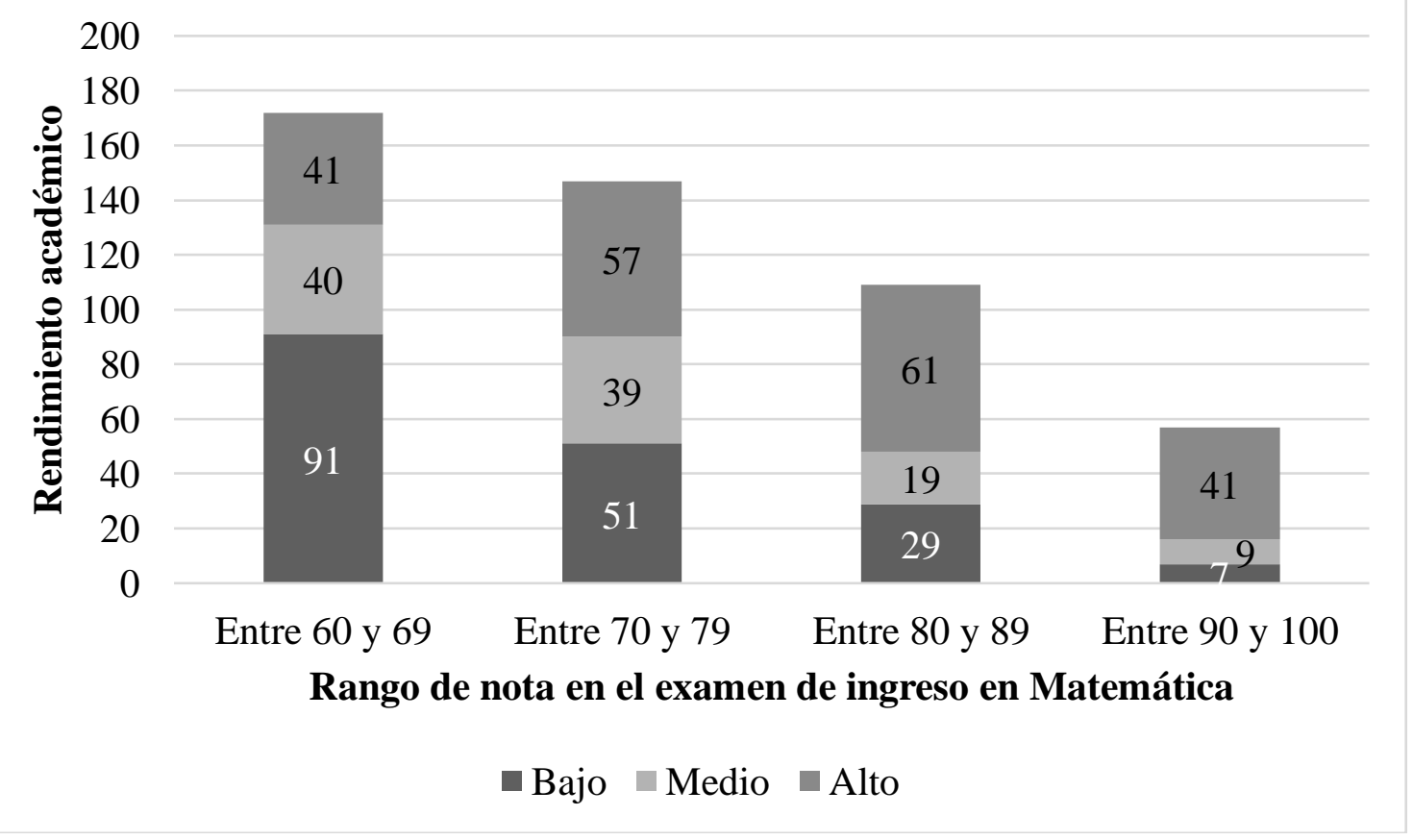

Figura 5. Gráfico del resultado de la tabulación cruzada entre el rango de notas obtenidas por los estudiantes en el EIM y el RAMP en el primer año de la carrera.

Nota: EI RAMP se consideró alto si tiene todas las asignaturas aprobadas, medio si tiene 1 o 2 asignaturas suspensas y bajo si suspende 3 o más asignaturas 
El 52\% (91) de los estudiantes que tienen notas en el EIM por debajo de 70 puntos, presentan bajo RAMP al culminar el primer año de estudios. Mientras el $72 \%$ (41) de los estudiantes que tienen notas en el EIM por encima de 90 puntos, presentan un alto RAMP, o sea, todas las asignaturas aprobadas.

Con respecto a la $\mathrm{H} 3$ que relaciona las variables RAMP y la deserción estudiantil, de la Figura 6 se puede decir que $93(52 \%)$ de los estudiantes de bajo RAMP causan baja durante el primer año, mientras que 85 estudiantes (48\%) repiten el año académico. Los 93 estudiantes que causaron baja y tienen bajo rendimiento, representan el $52 \%$ de los que tienen bajo rendimiento y el $96 \%$ de los que causaron baja.

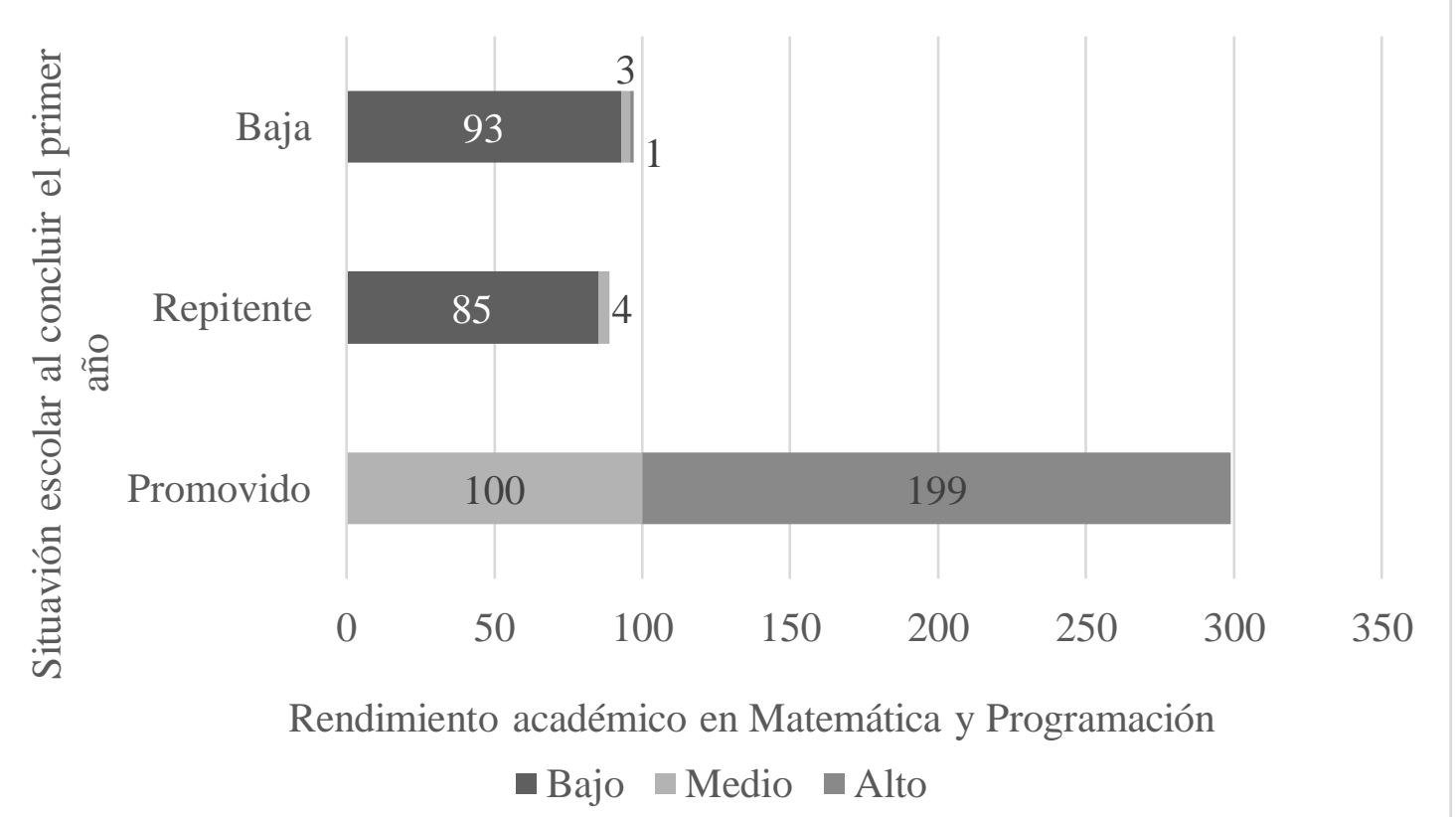

Figura 6. Gráfico del resultado de la tabulación cruzada entre el RAMP y la Deserción Estudiantil (Situación escolar al concluir el primer año)

La correlación permite concluir que en la medida que aumenta el rendimiento académico, disminuye la deserción estudiantil ya que se obtiene una alta correlación inversa de Spearman $R_{s}(8)=-.873^{\star *}, \mathrm{p}<.01$ y $N=$ 485.

La H4 relaciona la repitencia de un año académico y la deserción estudiantil en carreras de Ingeniería Informática. De la tabulación cruzada de los datos se infiere que de los 485 estudiantes que ingresaron en el curso 2013-2014, 89 repitieron en primer año académico. De estos 89 estudiantes, 36 (40\%) causaron baja durante el curso 2014-2015 o se cambiaron de carrera y 53 promovieron al segundo año. Asimismo, se comprueba que no existe relación entre dichas variables pues rs $(1)=.052, p>05$.

Del análisis anterior se puede concluir que la provincia de procedencia, la opción en que solicitó la carrera, la nota del EIM y el RAMP son factores que inciden en la deserción estudiantil.

\subsection{Análisis de regresión logística}

Para determinar los indicadores predictivos se realizó un análisis de regresión logística $(R L)$ cuyos resultados se resumen a continuación, indicando solo las variables que resultaron predictivas.

Las variables predictivas identificadas para $\mathrm{H} 1$ mediante $\mathrm{RL}$ binaria son la nota en el EIM y que procedan de alguna de las provincias siguientes: Las Tunas, Ciego de Ávila, Sancti Spíritu, Villa Clara, Pinar del Rio y Camagüey. La mayor probabilidad para que un estudiante cause baja es que tenga 60 puntos en el EIM y sea de la Provincia Camagüey. Con la función obtenida, se clasifica correctamente el $80 \%$ de los estudiantes.

En el caso de la $\mathrm{H} 2$ se ha utilizado RL multivariada pues la variable dependiente RAMP no es dicotómica, toma tres valores: alto, medio o bajo como muestra la Figura 6 . En este análisis se obtuvo que el RAMP bajo y medio se pueden predecir a partir de la nota en el EIM.

En el análisis de la H3 donde se relaciona el RAMP con la deserción, 93 estudiantes que causaron baja, tuvieron bajo RAMP, esto representa el $52 \%$ de los que tienen bajo rendimiento y el $96 \%$ de los que causaron 
baja. Se obtiene que la variable RAMP es predictiva para la deserción, la probabilidad de que un estudiante deserte o cause baja cuando su rendimiento es bajo es $p=.99$.

En el caso de H4, las variables repitencia del primer año y la deserción estudiantil, del apartado anterior se obtuvo que no existe correlación entre estas variables. En correspondencia con ello, el análisis de regresión entre dichas variables $(\beta=20.077, p>.05)$ por lo que no es predictiva la variable repitencia del primer año para la deserción estudiantil y se rechaza la $\mathrm{H} 4$.

El análisis de regresión realizado permitió identificar como variables predictivas de deserción estudiantil en el primer año de carreras de perfil Ingeniería Informática, el RAMP, la nota del EIM y la provincia de procedencia. Asimismo se infiere que la nota del EIM puede predecir el RAMP al culminar el primer año.

\subsection{Análisis discriminante}

El análisis discriminante se realizó con dos objetivos: (i) identificar las variables que discriminan o diferencian los estudiantes en los que desertan de la carrera, repiten el primer año académico o promueven, (ii) determinar cuáles son las variables que mejor explican la pertenencia de un estudiantes a uno de los tres grupos a partir de la función discriminante capaz de clasificar de forma más precisa a los estudiantes en estos tres grupos, a partir de conocer los datos de las variables discriminantes: X1=nota del EIM, X2=opción en que solicitó la carrera y X3=RAMP.

Para ello se tuvo en cuenta que los rangos y logaritmos naturales de los determinantes de cada grupo son cercanos entre sí. Asimismo, la prueba de igualdad de medias de grupos es significativa con $\mathrm{p}<.05$ para las tres variables discriminantes, por lo que se infiere que existe diferencia entre los grupos y es posible clasificar los estudiantes en los diferentes grupos (baja, repitentes y promovidos).

La correlación canónica de la función 1 es muy alta .923 y la prueba Lambda de Wilks está más cercana a cero .146, lo que indica que las variables discriminantes permiten diferenciar entre los grupos y dicha función es capaz de separar mejor los datos. La función 1 es capaz de explicar el $99.9 \%$ de la varianza de los datos y el valor $\mathrm{X}_{2}(6, N=485)=924.334, \mathrm{p}<.01$ por lo que es significativa.

Se escogen los coeficientes aportados para la función 1, pues en la Figura 7 se aprecia el poder de clasificación de los tres grupos de estudiantes en el eje de las abscisas. Para la función 1 los centroides de cada grupo están más alejados entre sí.

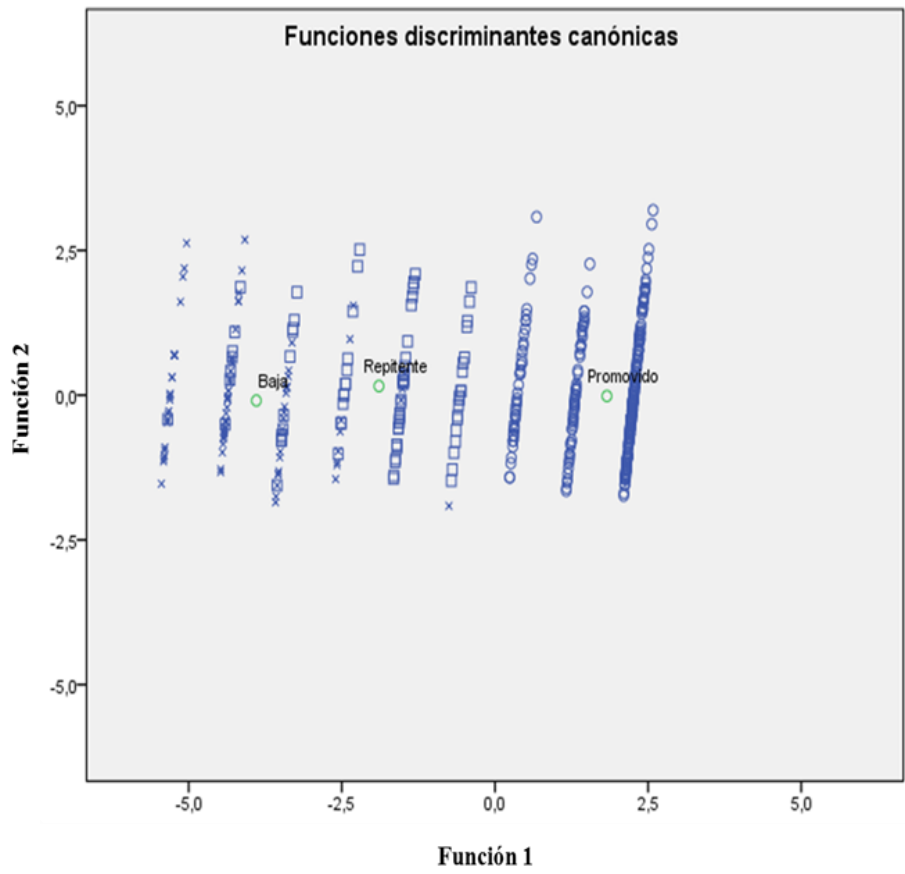

$$
\begin{aligned}
& \text { Promovido } \\
& \square \text { Repitente } \\
& \text { X Baja } \\
& \text { Centroides de grupos }
\end{aligned}
$$

Función 1

Figura 7. Representación del diagrama de dispersión de las Funciones Discriminantes 1 y 2 por grupos de clasificación. 
En base a los resultados de clasificación en la Tabla 8, se puede concluir que el análisis discriminante permite clasificar el $91.5 \%$ de los casos correctamente.

\section{Tabla 8}

Clasificación pronosticada respecto a la SE al cerrar el primer año.

\begin{tabular}{llcccc}
\hline & & \multicolumn{2}{c}{ Pertenencia a grupos pronosticada } & \\
SE al cerrar primer curso & Promovido & Repitente & Baja & Total \\
\hline Recuento & Promovido & 299 & 0 & 0 & 299 \\
& Repitente & 4 & 64 & 21 & 89 \\
& Baja & 4 & 12 & 81 & 97 \\
$\%$ & Promovido & 100,0 & 0,0 & 0,0 & 100,0 \\
& Repitente & 4,5 & 71,9 & 23,6 & 100,0 \\
& Baja & 4,1 & 12,4 & 83,5 & 100,0 \\
\hline
\end{tabular}

En la Tabla 9 la exhaustividad indica que se pueden predecir el 81\% de los casos de deserción.

\section{Tabla 9}

Valor de Exactitud (accuracy) Exhaustividad (recall) Precisión (precision) y el valor $F$ (f-measure) de los casos clasificados como deserción.

\begin{tabular}{lc}
\hline Performance and validation measure & Value \\
\hline Accuracy & .91 \\
Recall & .81 \\
Precision & .78 \\
F-measure & .79 \\
\hline
\end{tabular}

De los casos clasificados como deserción, el $78 \%$ son exactamente estudiantes que desertan, los falsos positivos y negativos requieren un estudio más pormenorizado.

\section{Conclusiones}

Se ha presentado un estudio para identificar los factores relevantes que inciden en la deserción de los estudiantes universitarios de Ingeniería Informática en Cuba. Los modelos predictivos de deserción-retención de estudios universitarios de la literatura han seguido enfoques de tipo psicológico, sociológico, interaccionista, organizacional y economicista para identificar variables que permitan explicar la problemática, que puede estar relacionadas con cuestiones personales, familiares, institucionales y/o académicas que varían según el contexto y carrera que se analice. Las causas más comunes son el rendimiento académico, la motivación y la integración académica y social. Se identifica que son los estudiantes del primer año los de mayor deserción.

Una revisión sistemática de los artículos indexados en Web of Science nos ha permitido identificar que estos estudios en carreras de Ingeniería Informática son escasos. Se analizaron en detalle 24 estudios que sirvieron de base para seleccionar las variables a analizar en el contexto cubano. En ellos se introducen variables como competencias teóricas y prácticas, la preparación previa en Matemática y el desarrollo del pensamiento lógico y analítico por la importancia que estas tienen y la base que aportan al estudio de la Informática. También se introducen variables pedagógicas como son los métodos de enseñanza aprendizaje de la Matemática, la motivación por el estudio de dichas carreras y la integración profesional.

Del análisis descriptivo, correlacional y predictivo realizado con una muestra de estudiantes de nuevo ingreso de todas las provincias cubanas, a carreras de perfil Ingeniería Informática en la cohorte 2013-2014, se identificaron como factores que inciden en la deserción estudiantil la provincia de procedencia, la opción en que solicitó la carrera, la nota del examen de ingreso en Matemática (EIM) y el rendimiento académico en Matemática y Programación. Se ha obtenido asimismo una función discriminante capaz de clasificar correctamente el $91.5 \%$ de los casos estudiados.

Nuestro estudio en el contexto cubano corrobora algunos resultados de estudios previos generales y también arroja resultados novedosos. Con respecto a la variable nota en el EIM el resultado indica que esta es una 
variable muy importante tanto para el rendimiento académico en primer año como para el abandono, al parecer en cualquier entorno, coincidiendo con los resultados en otros contextos por diversos autores (Graffigna et al., 2014; Heublein \& Wolter, 2011; Kori et al., 2018; Niitsoo et al., 2014; Paivi, 2012; SalazarFernandez et al., 2019; Van Den Broeck et al., 2017) que las estudian acompañadas de otras como la situación financiera, motivacional, expectativas, entre otras.

La variable género no resultó predictiva de deserción en el contexto cubano lo que no coincide con el resultado de (Miliszewska et al., 2006) en el contexto australiano. Igual ocurre en el caso de la provincia de residencia como factor predictivo, esta variable fue estudiada por (Lacave et al., 2018) y no resultó predictiva dentro del perfil de abandono obtenido.

Este estudio permitirá aportar acciones que desde la institución y el proceso docente educativo se pueden realizar para atender estos factores en el contexto cubano. Se espera que este estudio sirva de referencia a otros investigadores preocupados por esta temática pues aporta resultados que no tienen precedencia en este contexto.

\subsection{Limitaciones y recomendaciones para trabajo futuro}

El análisis se realizó con los datos de los estudiantes disponibles en el momento del estudio, en tal sentido, se considera una limitación el no contar con datos que involucren variables socioeconómicas y motivacionales. Asimismo, en los estudios de deserción analizados se identificaron más cuantitativos que cualitativos y pocos estudios mixtos, estos últimos para verificar convergencia de los datos y corroborar o no los resultados sobre las causas de la deserción.

Ambas limitaciones se resolverán en el trabajo futuro donde se ampliarán las variables en análisis, se incluirán un estudio de minería de datos y otro cualitativo de entrevistas semiestructuradas a los estudiantes que causaron baja en el primer año de la cohorte analizada. También se incorpora una profundización respecto a la incidencia de los mecanismos de admisión en la deserción. Finalmente, se realizará una propuesta de acciones tutoriales para atender los factores identificados.

\section{Referencias}

Ahmed, N., Kloot, B., \& Collier-Reed, B. I. (2015). Why students leave engineering and built environment programmes when they are academically eligible to continue. European Journal of Engineering Education, 40(2), 128-144. https://doi.org/10.1080/03043797.2014.928670

ALFA GUIA. (2011). Recuperado de http://www.alfaguia.org/www-alfa/index.php/es/

Altin, H., \& Rantsus, R. (2015). Why students fail to graduate ict-related curricula at university level. In INTED2015: 9th International Technology, Education and Development Conference (pp. 5364-5368). Madrid: IATED. Recuperado de https://sisu.ut.ee/sites/default/files/ict/files/heilo_altin_inted.pdf

Alzen, J. L., Langdon, L. S., \& Otero, V. K. (2018). A logistic regression investigation of the relationship between the Learning Assistant model and failure rates in introductory STEM courses. International Journal of STEM Education, 5(1), $1-12$.

https://doi.org/10.1186/s40594-018-0152-1

Araque, F., Roldán, C., \& Salguero, A. (2009). Factors influencing university drop out rates. Computers and Education, 53(3), 563-574.

https://doi.org/10.1016/j.compedu.2009.03.013

Arce, M. E., Crespo, B., \& Míguez, C. (2015). Higher Education Drop-Out in Spain - Particular Case of Universities in Galicia. International Education Studies, 8(5), 247-264.

https://doi.org/10.5539/ies.v8n5p247

Arriaga, J., Velásquez, M., \& Coord. (2013). Marco Conceptual sobre el Abandono. Construcción colectiva del concepto de abandono en la educación superior para su medición y análisis. Proyecto ALFA GUIA.

Barragán, S., \& González, L. (2015). Un modelo para explicar la retención en la Universidad de Bogotá Jorge Tadeo Lozano: arboles de decisión. V CLABES. Recuperado de

https://revistas.utp.ac.pa/index.php/clabes/article/view/1090/1113

Bean, J. P. (1980). Dropouts and turnover: The synthesis and test of a causal model of student attrition. Research in Higher Education, 12(2), 155-187. https://doi.org/10.1007/BF00976194

Bellei, C. coord. (2013). Situación Educativa de América Latina y el Caribe: OREALC-UNESCO.

Bernardo, A., Esteban, M., Fernández, E., Cervero, A., Tuero, E., \& Solano, P. (2016). Comparison of personal, social and academic variables related to university drop-out and persistence. Frontiers in Psychology, 7(OCT), 1-9.

https://doi.org/10.3389/fpsyg.2016.01610 
Bonaldo, L., \& Nobre, L. (2016). Dropout: Demographic profile of Brazilian university students. Procedia - Social and Behavioral Sciences, 228(June), 138-143.

https://doi.org/10.1016/j.sbspro.2016.07.020

Bulgarelli-Bolaños, R. M., Rivera-Rodríguez, J. A., \& Fallas-Vargas, M. A. (2017). El proceso vocacional del

estudiantado universitario en condición de logro y rezago académico : Un análisis desde el enfoque evolutivo de Donald Super. Revista Electrónica Educare, 21(1), 1-24.

https://doi.org/10.15359/ree.21-1.1

Cabrera, A. F., Nora, A., \& Castañeda, M. B. (1992). The role of finances in the persistence process: A structural model. Research in Higher Education, 33(5), 571-593.

https://doi.org/10.1007/BF00973759

Canedo, E. D., Santos, G. A., \& Leite, L. L. (2018). An Assessment of the Teaching-Learning Methodologies Used in the Introductory Programming Courses at a Brazilian University. Informatics in Education, 17(1), 45-59.

https://doi.org/10.15388/infedu.2018.03

Da Re, L., \& Clerici, R. (2017). Abandono, rendimiento académico y tutoría : una investigación de la Universidad de Padua. Educatio Siglo XXI, 35(2), 139-160.

https://doi.org/10.6018/j/298551

De Conincka, D., Matthijsa, K., \& Luyten, P. (2019). Subjective well-being among first-year university students: A twowave prospective study in Flanders, Belgium. Student Success, 10(1), 33-45.

https://doi.org/10.5204/ssj.v10i1.642

Díaz Peralta, C. (2008). Modelo conceptual para la desercion estudiantil universitaria chilena. Estudios Pedagógicos, XXXIV(2), 65-86. Recuperado de http://www.scielo.cl/pdf/estped/v34n2/art04.pdf

Donoso, S., \& Schiefelbein, E. (2007). Analisis de los modelos explicativos de retencion de estudiantes en la Universidad: una vision desde la desigualdad social. Estudios Pedagógicos, XXXIII(1), 7-27.

https://doi.org/10.4067/S0718-07052007000100001

Elster, D. (2014). First-Year Students' Priorities and Choices in STEM Studies - IRIS Findings from Germany and Austria. Science Education International, 25(1), 52-59. Recuperado de https://pdfs.semanticscholar.org/e2dc/0f12503f06ceedb7688170e057b8692dafd5.pdf

Estrada, R., Zaldivar, A., Nava, L., Peraza, J. F., Zaragoza, J. N., Diaz, E. M., \& Aguilar, C. (2011). Identification of variables associated with academic success of higher education students applying data mining. In A. Chova, LG; Belenguer, DM; Martinez (Ed.), 3rd International Conference on Education and New Learning Technologies (EDULEARN) (pp. 4958-4963).

Ethington, C. A. (1990). A psychological model of student persistence. Research in Higher Education, 31(3), $279-293$. https://doi.org/10.1007/BF00992313

Fonseca, G., \& García, F. (2016). Permanencia y abandono de estudios en estudiantes universitarios : un análisis desde la teoría organizacional. Revista de La Educación Superior, 45(179), 25-39.

https://doi.org/10.1016/j.resu.2016.06.004

García de Fanelli, A. (2018). Panorama de la educación superior en Iberoamérica. Red INDICES.

González-Rámirez, T., \& Pedraza-Navarro, I. (2017). Variables sociofamiliares asociadas al abandono de los estudios universitarios. Educatio Siglo XXI, 35(2), 365-388. https://doi.org/10.6018/j/298651

Graffigna, A. M., Hidalgo, L., Jofré, A., Berenguer, M. D. C., Moyano, A., \& Esteybar, I. (2014). Tutorial Practice as a Strategy of Retention at the School of Engineering. Procedia - Social and Behavioral Sciences, 116, 2489-2493. https://doi.org/10.1016/j.sbspro.2014.01.598

Hernández-Sampieri, R., Fernández-Collado, C., \& Baptista-Lucio, M. del P. (2014). Metodología de la Investigación. (McGRAW-HIL, Ed.) (Sexta). México. Recuperado de

https://periodicooficial.jalisco.gob.mx/sites/periodicooficial.jalisco.gob.mx/files/metodologia_de_la_investigacion_roberto hernandez sampieri.pdf

Hernández, D. de la C., Vargas, A., Almuiñas, J. L., \& García, J. L. (2015). Los indicadores actuales de la eficiencia académica: necesidad de su perfecionamiento. Pedagogía Universitaria, $X X(3), 53-62$.

Heublein, U., \& Wolter, A. (2011). Studienabbruch in Deutschland. Definition, Häufigkeit, Ursachen, Maßnahmen. Zeitschrift Für Pädagogik, 57(2), 214-236.

Horruitiner Silva, P. (2009). La Universidad Cubana: el Modelo de Formación. Editorial Universitaria del Ministerio de Educación Superior.

IESALC/UNESCO. (2006). Repitencia y deserción universitaria en América Latina (CINDA). Recuperado de https://www.cinda.cl/download/libros/Repitencia y Deserción Universitaria en América Latina.pdf Kori, K., Pedaste, M., \& Must, O. (2018). The Academic, Social, and Professional Integration Profies of Information Technology Students. ACM Trans. Comput. Educ., 18(4), 20:1-20:19.

https://doi.org/10.1145/3183343

Kuriakose, R. B. (2017). Freshman engineering student perceptions of academic feedback-a case study from Digital Systems 1. In 3rd International Conference on Higher Education Advances, HEAd'17 (pp. 42-54).

https://doi.org/10.1109/FIE.2016.7757443 
Lacave, C., Molina, A. I., \& Cruz-Lemus, J. A. (2018). Learning Analytics to identify dropout factors of Computer Science studies through Bayesian networks. Behaviour \& Information Technology, 0(0), 1-15.

https://doi.org/10.1080/0144929X.2018.1485053

López, S., Carpeño, A., Arriaga, J., Ruiz, M., \& Martín, A. (2016). Experiencias para el Fomento de las Vocaciones Tecnológicas entre Estudiantes de Enseñanza Secundaria. In A. Lago Ferreiro \& y M. G. Gericota (Eds.), TICAI 2016: TICs para el Aprendizaje de la Ingeniería. (pp. 89-96).

Maris, S., \& Difabio, H. (2009). Academic achievement and formal thought in engineering students. Electronic Journal of Research in Educational Psychology, 7(2), 653-672.

MES. Normas Generales del Sistema de Ingreso a la Educación Superior (2014). Recuperado de https://www.mes.gob.cu/sites/default/files/documentos/resoluciones/2014/Res 97.2014.pdf

MES. Reglamento de Organización Docente de la Educación Superior (2017). Cuba.

Meyer, M., \& Fang, N. (2019). A qualitative case study of persistence of engineering undergraduates. International Journal of Engineering Education, 35(1), 99-108.

Miliszewska, I., Barker, G., Henderson, F., \& Sztendur, E. (2006). The Issue of Gender Equity in Computer Science What Students Say. Journal of Information Technology Education, 5. Recuperado de https://cutt.ly/Kr8Fusz

Murtaugh, P. A., Burns, L. D., \& Schuster, J. (1999). Predicting the retention of university students. Research in Higher Education, 40(3), 355-371. Recuperado de https://link.springer.com/article/10.1023/A:1018755201899

Niitsoo, M., Paales, M., Pedaste, M., Siiman, L., \& Tõnisson, E. (2014). Predictors of informatics students ' progress and graduation in university studies. In Proceedings of INTED2014 Conference (pp. 2521-2529).

Paimin, A. N., Prpic, J. K., Hadgraft, R. G., \& Alias, M. (2017). Understanding student 's learning experiences in higher education. In Proceedings of INTED2017 Conference (pp. 6670-6676). Valencia, Spain.

Paivi, P. (2012). Enthusiasm Towards Mathematical Studies in Engineering. Proceedings of the 36th Conference of the International Group for the Psychology of Mathematics Education, 3(1990), 313-320. Recuperado de https://www.academia.edu/1869433/Enthusiasm_towards_Mathematical_Studies_in_Engineering

Pascarella, E. T., Pierson, C. T., Wolniak, G. C., \& Terenzini, P. T. (2004). First-Genēration College Students : Additional Evidence on College Experiences and Outcomes. The Journal of Higher Education, 75(3), 249-284.

https://doi.org/10.1353/jhe.2004.0016

Pascarella, E. T., \& Terenzini, P. T. (1980). Predicting Freshman Persistence and Voluntary Dropout Decisions from a Theoretical Model. The Journal of Higher Education, 51(1), 60-75. Recuperado de http://www.jstor.org/stable/1981125 Peña-Calvo, J. V., Inda-Caro, M., Rodríguez-Menéndez, C., \& Fernández-García, C. M. (2016). Perceived Supports and Barriers for Career Development for Second-Year STEM Students. Journal of Engineering Education, 105(2), $341-365$. https://doi.org/10.1002/jee.20115

Peña-Hernández, Y., Martínez-Sánchez, N., \& Jesús-Calderíus, M. de. (2019). Apuntes históricos sobre la permanencia estudiantil en el contexto universitario cubano. EduSol, 19(66), 98-112.

Portella Teixeira de Mello, S., de Melo, P. A., \& Teixeira de Mello Filho, R. (2015). Estudando a evasão no ensino tecnológico em uma instituição de ensino superior no sul do Brasil. EccoS - Revista Científica, (37), 181-196. https://doi.org/10.5585/eccos.n37.4890

Salazar-Fernandez, J. P., Sepúlveda, M., \& Munoz-Gama, J. (2019). Influence of Student Diversity on Educational Trajectories in Engineering High-Failure Rate Courses that Lead to Late Dropout. In IEEE Global Engineering Education Conference (EDUCON) (pp. 607-616).

SelecciónWos, (2019). http://lsi.ugr.es/zoraida/Fuentes20/articulos.html

Soistak, M. M., Martins, L. M., \& Galan, T. do C. (2018). Why engineering students give up in their formation : a case. Nuances: Estudos Sobre Educação, 29(1), 154-168.

https://doi.org/10.32930/nuances.v29i1.4391.154

Spady, W. G. (1970). Dropouts from Higher Education: An Interdisciplinary Review and Synthesis. Interchange, 1(1), 6485.

https://doi.org/10.1007/BF02214313

Suárez-Montes, N., \& Díaz-Subieta, L. B. (2015). Estrés académico, deserción y estrategias de retención de estudiantes en la educación superior. Revista de Salud Pública, 17(2), 300-313.

https://doi.org/10.15446/rsap.v17n2.52891

Tinto, V. (1975). Dropout from Higher Education: A Theoretical Synthesis of Recent Research. Review of Educational Research Winter, 45(1), 89-125.

Tinto, V. (2010). From theory to action: Exploring the institutional conditions for student retention. (J.C. Smart (ed.), Ed.) (Springer N). Higher education: Handbook of theory and research.

https://doi.org/10.1007/978-94-007-2950-6

Úbeda-Sánchez, Á. M., Fernández-Cano, A., \& Callejas, Z. (2019). Inferring hot topics and emerging educational research fronts. On the Horizon, 27(2), 125-134.

https://doi.org/10.1108/OTH-04-2019-0017

Van Den Broeck, L., De Laet, T., Lacante, M., Pinxten, M., Van Soom, C., \& Langie, G. (2017). Comparison between bridging students and traditional first-year students in engineering technology. European Journal of Engineering Education, $0(0), 1-16$.

https://doi.org/10.1080/03043797.2017.1417357 
Xenos, M., Pierrakeas, C., \& Pintelas, P. (2002). A survey on student dropout rates and dropout causes concerning the students in the Course of Informatics of the Hellenic Open University. Computers and Education, 39, 361-377. https://doi.org/10.1016/S0360-1315(02)00072-6

Yong-Min, L., Ai-bin, C., \& Jun, T. (2009). A new viewpoint on the reason of computer science course's students drop out. In ETP/ IITA World Congress in Applied Computing, Computer Science and Computer Engineering (pp. 185-188). Sanya, Peoples R China: ETP-Engineering Technology Press.

Zehetmeier, D., Axel, B., Bruggemann-Klein, A., Thurner, V., \& Schlierkamp, K. (2015). Finding Competence

Characteristics among First Semester Students in Computer Science. In 45th Annual Frontiers in Education Conference (pp. 1079-1087).

https://doi.org/10.1109/FIE.2015.7344201 